\title{
Molecular Remission at T cell level in Patients with Rheumatoid Arthritis
}

\author{
Jun Inamo \\ Keio University \\ Katsuya Suzuki \\ Keio University \\ Masaru Takeshita \\ Keio University \\ Yasushi Kondo \\ Keio University \\ Yuumi Okuzono \\ Takeda Pharmaceutical Co Ltd \\ Keiko Koga \\ Takeda Pharmaceutical Co Ltd \\ Yoshiaki Kassai \\ Takeda Pharmaceutical Co Ltd \\ Maiko Takiguchi \\ Takeda Pharmaceutical Co Ltd \\ Rina Kurisu \\ Takeda Pharmaceutical Co Ltd \\ Akihiko Yoshimura \\ Keio University \\ Tsutomu Takeuchi ( $\nabla$ tsutake@z5.keio.jp ) \\ Keio University
}

\section{Research Article}

Keywords: Rheumatoid arthritis, T cells, molecular remission

Posted Date: May 18th, 2021

DOI: https://doi.org/10.21203/rs.3.rs-51665/v3

License: (c) (1) This work is licensed under a Creative Commons Attribution 4.0 International License. Read Full License 
Version of Record: A version of this preprint was published at Scientific Reports on August 17th, 2021. See the published version at https://doi.org/10.1038/s41598-021-96300-z. 


\section{Abstract \\ Introduction.}

While numerous disease-modifying anti-rheumatic drugs (DMARDs) have brought about a dramatic paradigm shift in the management of rheumatoid arthritis (RA), unmet needs remain, such as the small proportion of patients who achieve drug-free status. The aim of this study was to explore key molecules for remission at the T cell level, which are known to be deeply involved in RA pathogenesis, and investigate the disease course of patients who achieved molecular remission (MR).

\section{Methods.}

We enrolled a total of 46 patients with RA and 10 healthy controls (HCs). We performed gene expression profiling and selected remission signature genes in $\mathrm{CD} 4^{+} \mathrm{T}$ cells and $\mathrm{CD} 8^{+} \mathrm{T}$ cells from patients with RA using machine learning methods. In addition, we investigated the benefits of achieving MR on disease control.

\section{Results.}

We identified 9 and 23 genes that were associated with clinical remission in $\mathrm{CD} 4^{+}$and $\mathrm{CD} 8^{+} \mathrm{T}$ cells, respectively. Principal component analysis (PCA) demonstrated that their expression profiling was similar to those in $\mathrm{HCs}$. For the remission signature genes in $\mathrm{CD} 4^{+} \mathrm{T}$ cells, the PCA result was reproduced using a validation cohort, indicating the robustness of these genes. A trend toward better disease control was observed during 12 months of follow-up in patients treated with tocilizumab in deep MR compared with those in non-deep MR, although the difference was not significant.

\section{Conclusion.}

We identified robust genes that represent remission status in $\mathrm{CD} 4^{+} \mathrm{T}$ cells using machine learning techniques. The current study will promote our understanding of the molecular mechanisms necessary to achieve deep remission during the management of RA.

\section{Trial registration:}

Not required.

\section{Introduction}


Rheumatoid arthritis (RA) is an autoimmune disease characterized by chronic inflammation of the synovial tissue [1]. The advent of disease-modifying anti-rheumatic drugs (DMARDs) has brought about a dramatic paradigm shift in the management of RA. Currently, the goal for RA treatment is to achieve clinical remission (CR), which has been facilitated by the development of various types of biological agents. However, unmet needs in the management of RA remain for a large proportion of patients with remission. More than half of patients with CR experience an RA flare following DMARD cessation [2, 3]. Moreover, the existence of patients with difficult-to-treat RA [4], who ultimately become resistant to multiple types of DMARDs, introduces the need to understand the molecular status of remission.

Thus, molecular remission (MR) has been proposed to distinguish patients with "deep" remission from others $[5,6]$. A proteome study demonstrated that a low multi-biomarker disease activity score, which the researchers developed, was associated with limited radiographic progression over the following 12 months [5]. We recently investigated molecular signatures that were associated with deep remission at the multi-omics level [6]. In the previous report, drug treatments altered the molecular profile to better resemble that of healthy controls ( $\mathrm{HCs}$ ) at the transcriptomic, serum proteomic, and immunophenotypic levels. In addition, longitudinal monitoring suggested that the achievement of MR by DMARDs was associated with long-term stable CR. However, how each transcriptomic remission signature molecule is related to clinical traits remained unclear because we used whole-blood specimens, and the expression profiles varied according to cell subsets, indicating the necessity of further study using each cell subpopulation [6].

T cells are well-known to contribute to the pathogenesis of RA. Susceptible genes for RA outside the major histocompatibility complex locus are highly expressed in $\mathrm{CD} 4^{+} \mathrm{T}$ cells $[7,8]$. The evidence that a subset of $\mathrm{CD} 8^{+} \mathrm{T}$ cells is also critical a contributor to the development of RA is accumulating. $\mathrm{CD} 8^{+} \mathrm{T}$ cells are required for the development of ectopic germinal centers in the synovium, which is considered to be the home of the core immune response in RA [9]. Recently, to investigate and clarify the comprehensive characteristics of T cells in RA, we conducted a multi-dimensional, immunophenotyping analysis according to the developmental stage: $\mathrm{CD} 4^{+} \mathrm{T}$ cells were classified into four subsets, naïve (TN), stem cell memory (TSCM), central memory (TCM), and effector memory (TEM), whereas $C D 8^{+} \mathrm{T}$ cells were classified into five stages, TN, TSCM, TCM, TEM, and CD45RA-positive effector memory (TEMRA). The study demonstrated that the CD8 ${ }^{+}$TEMRA subset increased in patients with RA compared with HCs, and TEM-follicular helper (Tfh) cells and TEM-T helper 17(Th17) cells were correlated with disease activity, suggesting that T cells in patients with remission may represent the MR state of RA [10].

Here, we report the key molecules associated with remission at the T cell level and investigate the disease course of patients who achieved MR.

\section{Methods}

\section{Patients and control subjects}


The current study utilized cohorts from our previous report [10]. The detailed information was described previously. Briefly, 2 cohorts were included in this study (Cohort 6 and Cohort 7 of the previous report, Supplementary Table S1-2). Cross-sectional gene expression profiling was performed in the derivation cohort, and longitudinal gene expression profiling was performed at pre-treatment and post-treatment time points in the validation cohort (Fig. 1). Patients in the validation cohort were treated with tocilizumab (TCZ) for 6 months and achieved remission after treatment. Peripheral blood samples and synovial fluid (SF) samples were collected before treatment from patients with drug-naïve RA and during treatment from other patients, respectively. We separated each T cell subpopulation from the specimens, and RNA was isolated. Then, we conducted RNA-sequencing and performed bioinformatic analysis. Read count normalization was performed using the transcripts per million (TPM) method [13]. Disease activity was assessed by standard composite indices, such as DAS28-ESR (disease activity score 28-ESR) and DAS28-CRP, and remission was defined as a DAS28-CRP $<2.4$ [14]. This study was approved by the Institutional Review Board of Keio University School of Medicine and conducted according to the Declaration of Helsinki. Consent to participate was obtained from all subjects in the current study before blood specimen was collected.

\section{Development of RA remission signature models}

To select genes that had a strong relationship with the remission, we applied the least absolute shrinkage and selection operator (lasso) to normalized gene expression data of patients with drug-naïve RA and treatment in the derivation cohort. Lasso is a machine learning method that is suitable for detecting key variables without prior feature selection from a multivariate dataset that contains only a few covariates that are associated with the outcome, which improves the prediction accuracy and interpretability of regression models [15]. To avoid overfitting, we first divided the gene expression dataset of the derivation cohort into a training dataset and a test dataset (with ratio $=7: 3$ ) and conducted lasso using the training dataset with seven-fold cross-validation. Then, a partial least-squares regression (PLS-R) was utilized to weight the values of selected genes and construct a model that was useful for separating remission and non-remission patients. The variable importance in projection (VIP) score obtained by PLS-R is a significant measurement for each predictor variable. Genes with VIP scores greater than 1 were considered to be related to remission [16]. Thus, we can evaluate the importance of each gene. Receiver operating characteristic (ROC) analysis was used to evaluate the prediction accuracy of the test data using the statistical model generated from the training set. Then, the statistical model was applied to the whole dataset to enumerate the "remission odds" for each sample. The caret and ropls R package were used for lasso and PLS-R modeling, respectively [17].

\section{Principal component analysis and pathway mapping}

Principal component analysis (PCA) was performed using normalized data with the FactoMineR $\mathrm{R}$ package [18]. To gain a functional annotation of selected genes, Enrichr's plugin [19] KEGG pathways [20] was used.

\section{Statistics}


Continuous data are presented as the median and interquartile range or as a number and percentage, as appropriate. The Wilcoxon rank-sum test was used to examine differences between continuous variables. Fisher's exact test was used to compare proportions in categorical data between groups. All statistical analyses were performed with R (R Foundation for Statistical Computing, Vienna, Austria).

\section{Results}

\section{Remission signature genes in RA}

The primary objective was to explore genes that are relevant to remission status in RA (referred to as remission signature genes). Therefore, we first selected genes that were useful for separating patients with remission from those with non-remission using a training dataset from the derivation cohort, separated according to $\mathrm{CD} 4^{+}$and $\mathrm{CD} 8^{+} \mathrm{T}$ cells. Using lasso on all 15,304 transcripts, 17 and 46 genes were selected as important molecules in $\mathrm{CD} 4^{+}$and $\mathrm{CD} 8^{+} \mathrm{T}$ cells, respectively, to classify patients with remission from those with non-remission.

Then, we weighted the genes selected by lasso and constructed a statistical model to separate remission from non-remission (referred to as the RA remission signature model) by applying PLS-R. The ROC analysis applied to the test set that was not used for training was separated able to separate the two populations with good accuracy (area under the curve [AUC], 0.947 and 0.929 for $C D 4^{+}$and CD $8^{+} \mathrm{T}$ cells, respectively; Fig. 2A). This result indicated that the combination of lasso and PLS-R captured informative genes from our data. In addition, 9 (e.g., MST1, ASB2, SULT2B1, and SOCS3) and 23 (e.g., CRLF2, NIM1, and ID1) genes met the criteria (VIP >1) for model inclusion in $\mathrm{CD} 4^{+}$and $\mathrm{CD} 8^{+} \mathrm{T}$ cells, respectively (Fig. 2B and C and Supplementary Table S3). Hereafter, we refer to these genes as remission signature genes. To understand the function of remission signature genes, pathway analysis was performed (Fig. 2B and C). In CD4 ${ }^{+} \mathrm{T}$ cells, molecules involved in various metabolic pathways (Vitamin B6 metabolism and Glycine, serine, and threonine metabolism), endocrine pathways (steroid hormone biosynthesis, adipocytokine signaling pathway, prolactin signaling pathway, and insulin resistance), and the TNF signaling pathway were enriched. In $\mathrm{CD}^{+} \mathrm{T}$ cells, molecules involved in metabolic pathways (taurine and hypotaurine metabolism and fatty acid degradation) and the JAK-STAT signaling pathway were enriched.

\section{Remission odds of each T cell subpopulation}

To investigate the effects of different DMARDs and on remission signature genes in the various $T$ cell subpopulations (TN, TCM, TEM, and TEMRA), we compared remission odds according to each subgroup. Using the RA remission signature model generated by the combination of lasso and PLS-R, the remission odds of each subject were produced: if patients were in remission status, remission odds were $>0.5$, whereas if patients were far from remission, remission odds were $<0.5$. Because T cells in SF are 
considered to reflect pathological status, we calculated the remission odds of the T cell subpopulation in SF from some patients.

In $C D 4^{+} \mathrm{T}$ cells, the remission odds of patients with DMARDs, all of whom were in remission, except for one patient (Fig. 1), were significantly higher compared with those of drug-naïve patients (Fig. 3A). Of note, although significance was not achieved due to limited samples, SF samples trended toward low remission odds, as did drug-naïve samples from peripheral blood, suggesting that remission signature genes might represent the pathogenic status of RA. Compared with HCs, the DMARD-treated patients had similar values, regardless of drug type, indicating that all drugs pushed the pathogenic gene expression profile of remission signature genes toward the healthy state. Correspondingly, PCA using remission signature genes in $\mathrm{CD} 4^{+} \mathrm{T}$ cells demonstrated that only drug-naïve samples clustered from the other groups (Fig. 3B). To validate the classification ability of remission signature genes, PCA analysis was conducted using expression data from the validation cohort. Similar to earlier results, samples from patients in remission created clusters apart from those in non-remission, supporting the robustness of remission signature genes in $\mathrm{CD} 4^{+} \mathrm{T}$ cells (Fig. $3 \mathrm{C}$ ).

In the $\mathrm{CD} 8^{+} \mathrm{T}$ cell subpopulation, similar to $\mathrm{CD} 4^{+} \mathrm{T}$ cells, all remission odds of patients with DMARDs were significantly higher than those of drug-naïve patients (Fig. 4A). However, the remission odds of some samples in groups of DMARDs were also significantly higher than those of HCs, suggesting that selected genes in $\mathrm{CD}^{+} \mathrm{T}$ cells might not correctly represent the healthy state. In addition, PCA demonstrated that all clusters overlapped, except the TEM subpopulation (Fig. 4B). Further, PCA using validation cohorts was unable to validate the selected genes, indicating the vulnerable ability of remission signature genes in $\mathrm{CD}^{+} \mathrm{T}$ cells (Fig. 4C).

\section{Relations between molecular remission and following disease activity}

To elucidate the benefits of MR, we next addressed whether any differences existed between patients in "deep" MR and non-deep MR. To achieve this goal, we conducted a follow-up study of 29 consecutive patients (MTX, $n=10 ;$ IFX, $n=10 ; T C Z, n=9$ ) treated with DMARDs in the derivation cohort for up to 12 months after the measurement of gene expression. We defined the MR of each cell subset as remission odds greater than the average value of the remission odds for each cell subset, and deep MR was defined as each patient with greater than 4 cell subsets in MR (maximum 7). Of the 29 patients treated with DMARDs, 12 and 17 patients were classified as deep MR and non-deep MR. Disease activity was not significantly different at any time point (Fig. 5A). However, the cumulative DAS28-ESR (described as the AUC) of patients treated with TCZ in deep MR had a trend lower than those treated with TCZ in non-deep MR (12.48 [11.25-13.82] vs. 18.26 [17.07-18.36], $p=0.19$; Fig. 5B). Comparisons among drug types showed a significant difference between patients treated with TCZ and those treated with MTX in deep MR. Although we conducted sensitivity analysis by changing the outcome (e.g., DAS28-CRP and each component of the DAS28) and the definition of deep MR (e.g., the cut-off number of the cell 
subpopulation, limited to the $\mathrm{CD} 4^{+} \mathrm{T}$ cell subpopulations), we could not find significant benefit for deep MR in our data (data not shown).

\section{Discussion}

In the current study, we identified remission signature genes associated with RA in $\mathrm{CD} 4^{+}$and $\mathrm{CD} 8^{+} \mathrm{T}$ cells. Although those in $\mathrm{CD} 8^{+} \mathrm{T}$ cells were vulnerable when applied to another cohort, those in $\mathrm{CD} 4^{+} \mathrm{T}$ cells had the robust ability to classify remission and non-remission patients in both cross-sectional and longitudinal cohorts. Furthermore, the remission odds calculated by the RA remission model in $\mathrm{CD} 4^{+} \mathrm{T}$ cells showed similar value among patients in remission and HCs, suggesting that the 9 selected genes represent whether the $C D 4^{+} T$ cells of RA are pushed back to a healthy state. In addition, deep MR by TCZ had the potential to be associated with better disease control.

As shown in previous reports, $T$ cells were involved in the development and chronicity of RA [7-10]. Compared with $\mathrm{CD} 4^{+} \mathrm{T}$ cells, evidence supporting a role for $\mathrm{CD} 8^{+} \mathrm{T}$ cells in $\mathrm{RA}$ is still emerging [9]. Clonal expansion was observed for $\mathrm{CD} 8^{+} \mathrm{T}$ cells but not for $\mathrm{CD} 4^{+} \mathrm{T}$ cells in newly diagnosed patients with $\mathrm{RA}$, indicating that $C D 8^{+} T$ cells are necessary for the initial phase of RA [21]. However, RA risk alleles were preferentially expressed in $\mathrm{CD} 4^{+} \mathrm{T}$ cells but not in $\mathrm{CD} 8^{+} \mathrm{T}$ cells $[7,8]$, and only the $\mathrm{CD} 4^{+} \mathrm{T}$ cell subpopulation was positively associated with disease activity [10], suggesting that $\mathrm{CD} 4^{+} \mathrm{T}$ cells more deeply contribute to the activity state of RA than $\mathrm{CD} 8^{+} \mathrm{T}$ cells.

Remission signature genes identified in $\mathrm{CD} 4^{+} \mathrm{T}$ cells were downregulated in patients with remission. MST1 promotes the migration of T cells via the activation of LFA-1 [22, 23]. In addition, MST1-deficient T cells are prone to apoptosis [24]. ASB2 is known to promote NF-KB activation, leading to the suppression of T cell apoptosis [25]. SULT2B1 is involved in cholesterol homeostasis and is expressed in activated T cells, prompting proliferation via the inhibition of LXR signaling [26]. SOCS3 inhibits STAT3, a downstream molecule in the JAK-STAT pathway that is inhibited by TNFa- and IL-6 inhibition [27]; therefore, the downregulation of SOCS3 may lead to the activation of the inflammatory pathway [28]. However, the loss of SOCS3 in CD4 ${ }^{+} \mathrm{T}$ cells promotes anti-inflammatory cytokines, such as interleukin 10 and transforming growth factor-beta 1, and suppresses inflammatory responses [29], suggesting that the decreased expression of SOCS3 itself may be beneficial for controlling RA. Among the other 5 genes identified as remission signature genes in $C D 4^{+} \mathrm{T}$ cells (ABHD11, KCNK1, PSAT1, CYB5D2, and CABP4), their functions in $T$ cells remain unknown, and further functional study is needed to clarify their significance in RA.

However, we were unable to show any significant benefit for clinical course associated with achieving deep MR, as defined by remission signature genes, although those treated with TCZ in deep MR had a favorable trend. To date, although several studies have attempted to predict changes in disease activity using only transcriptome data, they have failed to show robust predictability [6,30]. In our data, most patients, even non-deep MR patients, were under good control during follow-up, as shown in Fig. 5A, 
which might make the detection of significant differences challenging. In addition, the definition of "deep" MR used in the current study lacks supporting evidence. Therefore, to explore the impact of MR on clinical traits and the associations between MR and drug types, we need to plan a prospective study with larger sample size.

This study suffers from several limitations. First, we did not examine the association between functional subpopulations and MR. We previously reported that Tfh and Th17 cells in TEM were correlated with disease activity [10]. In addition, the proportion of T peripheral helper cells, which act as pathogenic CD4 helper $T$ cells in RA, is associated with disease activity and treatment [31]. These findings suggest that the extent of $\mathrm{T}$ cell contributions to disease status varies and indicates the necessity of exploring remission signature genes according to their functions [10]. Second, the functions of these genes in T cells have not been investigated. In vitro or in vivo studies remain necessary to determine whether these genes are potential targets for therapy. Third, other potential benefits of MR were not considered in the current study, such as a lower risk of flares. Because the concept of MR in RA has only emerged recently, we need to validate the definition and effects of MR on real-world patients in future research using larger cohorts.

\section{Conclusions}

We identified robust remission signature genes in $\mathrm{CD} 4^{+} \mathrm{T}$ cells. The current study will highlight the utility of using transcriptome data in $\mathrm{CD} 4^{+} \mathrm{T}$ cells to classify remission and non-remission in RA and promote the development of novel therapeutic targets against RA.

\section{Abbreviations}

area under the curve (AUC); CD45RA-positive effector memory T cells (TEMRA); central memory T cells (TCM); clinical remission (CR); disease activity score-28 ESR (DAS28-CRP); disease activity score-28 ESR (DAS28-ESR); disease-modifying anti-rheumatic drugs (DMARDs); effector memory T cells (TEM); healthy controls (HCs); infliximab (IFX); least absolute shrinkage and selection operator (lasso); methotrexate (MTX); molecular remission (MR); naïve T cells (TN); partial least-squares regression (PLS-R); principal component analysis (PCA); Receiver operating characteristics (ROC); rheumatoid arthritis (RA); stem cell memory T cells (TSCM); synovial fluid (SF); T helper 17 cell (Th17); T-follicular helper cells (Tfh); tocilizumab (TCZ); transcripts per million (TPM); Variable importance in projection (VIP).

\section{Declarations}

\section{Ethics approval and consent to participate}

Ethics approval was obtained from the Institutional Review Board of Keio University School of Medicine. Consent to participate was obtained from all subjects in the current study before blood specimen was collected. 


\section{Consent for publication}

Not applicable.

\section{Availability of data and materials}

Transcriptome data are available at the GEO database. The accession codes are GSE113156 and GSE118829. All custom computer codes in the generation or processing of the described data are available upon reasonable request.

\section{Competing interests}

YO, KK, YK, MT and RK are employees of Takeda Pharmaceutical Company Limited. KS has received research grants from Eisai, Bristol-Myers Squibb, Kissei Pharmaceutical, and Daiichi Sankyo, and speaking fees from Abbie Japan, Astellas Pharma, Bristol-Myers Squibb, Chugai Pharmaceutical, Eisai, Fuji Film Limited, Janssen Pharmaceutical, Kissei Pharmaceutical, Mitsubishi Tanabe Pharmaceutical, Pfizer Japan, Shionogi, Takeda Pharmaceutical, and UCB Japan, consulting fees from Abbie, and Pfizer Japan. AY has received speaking fees from Chugai Pharmaceutical, Mitsubishi Tanabe Pharmaceutical, Pfizer Japan, Ono Pharmaceutical, Maruho, and Novartis, and consulting fees from GSK Japan. TT has received research grants from Astellas Pharma Inc, Bristol-Myers KK, Chugai Pharmaceutical Co. Ltd., Daiichi Sankyo Co. Ltd, Takeda Pharmaceutical Co. Ltd, Teijin Pharma Ltd, AbbVie GK, Asahikasei Pharma Corp, Mitsubishi Tanabe Pharma Co, Pfizer Japan Inc, and Taisho Toyama Pharmaceutical Co. Ltd, Eisai Co. Ltd, AYUMI Pharmaceutical Corporation, and Nipponkayaku Co. Ltd, and speaking fees from AbbVie GK, Bristol-Myers KK, Chugai Pharmaceutical Co. Ltd, Mitsubishi Tanabe Pharma Co, Pfizer Japan Inc, and Astellas Pharma Inc, and Diaichi Sankyo Co. Ltd, and consultant fees from Astra Zeneca KK, Eli Lilly Japan KK, Novartis Pharma KK, Mitsubishi Tanabe Pharma Co, Abbivie GK, Nipponkayaku Co. Ltd, Janssen Pharmaceutical KK, Astellas Pharma Inc, and Taiho Pharmaceutical Co. Ltd. JI, MT and YK declare no potential conflict of interest.

\section{Funding}

This work was supported by Takeda Pharmaceutical Company Limited, Kanagawa, Japan (grant number 04-078-0067) and Keio University (grant number 04-013-0188). 


\section{Authors' Contributions}

Conceptualization: JI, KS and MT. Funding acquisition: AY and TT. Data acquisition: MT, YK, KK, YK, MT and RK. Formal analysis: JI and YO. Supervision: KS. Writing and original draft preparation: JI. Writing review and editing: KS and TT.

\section{Acknowledgements}

We thank Ms. Harumi Kondo, Ms. Mayumi Ota, Ms. Yoshiko Yogiashi, Mr. Yuki

Otomo, Mr. Fumitsugu Yamane and Ms. Miku Shimizu for helping with the experiments. In addition, we thank Cambridge English Correction Service for proofreading sentences for English proofreading.

\section{References}

1. Mclnnes, I. B. \& Schett, G. The pathogenesis of rheumatoid arthritis. N Eng/ J Med. 365, 2205-2219 (2011).

2. Baker, K. F. et al. Predicting drug-free remission in rheumatoid arthritis: A prospective interventional cohort study. J Autoimmun. 105, 102298 (2019).

3. Kuijper, T. M. et al. Flare rate in patients with rheumatoid arthritis in low disease activity or remission when tapering or stopping synthetic or biologic DMARD: a systematic review. J Rheumatol. 42, 2012-2022 (2015).

4. Roodenrijs, N. M. T. et al. Characteristics of difficult-to-treat rheumatoid arthritis: results of an international survey. Ann Rheum Dis. 77, 1705-1709 (2018).

5. van der Helm-van Mil, A. H. et al. An evaluation of molecular and clinical remission in rheumatoid arthritis by assessing radiographic progression. Rheumatology (Oxford). 52, 839-846 (2013).

6. Tasaki, S. et al. Multi-omics monitoring of drug response in rheumatoid arthritis in pursuit of molecular remission. Nat Commun. 9, 2755 (2018).

7. Farh, K. K. H. et al. Genetic and epigenetic fine mapping of causal autoimmune disease variants. Nature. 518, 337-343 (2015).

8. $\mathrm{Hu}, \mathrm{X}$. et al. Integrating autoimmune risk loci with gene-expression data identifies specific pathogenic immune cell subsets. The American Journal of Human Genetics. 89, 496-506 (2011).

9. Kang, Y. M. et al. CD8 T cells are required for the formation of ectopic germinal centers in rheumatoid synovitis. J Exp Med. 195, 1325-1336 (2002).

10. Takeshita, M. et al. Multi-dimensional analysis identified rheumatoid arthritis-driving pathway in human T cell. Ann Rheum Dis. 78, 1346-1356 (2019).

11. Aletaha, D. et al. 2010 rheumatoid arthritis classification criteria: an American College of Rheumatology/European League Against Rheumatism collaborative initiative. Ann Rheum Dis. 69, 
1580-1588 (2010).

12. Saag, K. G. et al. American College of Rheumatology 2008 recommendations for the use of nonbiologic and biologic disease-modifying antirheumatic drugs in rheumatoid arthritis. Arthritis Rheum. 59, 762-784 (2008).

13. Li, B. et al. RNA-Seq gene expression estimation with read mapping uncertainty. Bioinformatics. 26, 493-500 (2010).

14. Fleischmann, R. et al. How much does Disease Activity Score in 28 joints ESR and CRP calculations underestimate disease activity compared with the Simplified Disease Activity Index? Ann Rheum Dis. 74, 1132-1137 (2015).

15. Tibshirani, R. The lasso method for variable selection in the Cox model. Stat Med. 16, 385-395 (1997).

16. Noppamas, A., Seree, C. \& Kidakan, S. Cutoff threshold of variable importance in projection for variable selection. Int J Pure Appl Math. 94, 307-322 (2014).

17. Thévenot, E. A. et al. Analysis of the Human Adult Urinary Metabolome Variations with Age, Body Mass Index, and Gender by Implementing a Comprehensive Workflow for Univariate and OPLS Statistical Analyses. J Proteome Res. 14, 3322-3335 (2015).

18. Lê, S., Josse, J., Husson, F. \& FactoMineR An R Package for Multivariate Analysis. J Stat Softw. 25, $1-18$ (2008).

19. Chen, E. Y. et al. Enrichr: interactive and collaborative HTML5 gene list enrichment analysis tool.BMC Bioinformatics. 2013;128(14).

20. Kanehisa, M. \& Goto, S. K. E. G. G. Kyoto Encyclopedia of Genes and Genomes. Nucleic Acids Res. 28, 27-30 (2000).

21. Savola, P. et al. Somatic mutations in clonally expanded cytotoxic T lymphocytes in patients with newly diagnosed rheumatoid arthritis.Nat Comms2017;8.

22. Katagiri, K., Imamura, M. \& Kinashi, T. Spatiotemporal regulation of the kinase Mst1 by binding protein RAPL is critical for lymphocyte polarity and adhesion. Nat Immunol. 7, 919-928 (2006).

23. Ueda, Y. et al. Mst1 regulates integrin-dependent thymocyte trafficking and antigen recognition in the thymus. Nat Commun. 3, 1098 (2012).

24. Choi, J. et al. Mst1-FoxO signaling protects Naïve T lymphocytes from cellular oxidative stress in mice. PLoS One. 4, e8011 (2009).

25. Wu, W. et al. The notch pathway promotes NF-kB activation through Asb2 in T cell acute lymphoblastic leukemia cells. Cell Mol Biol Lett. 23, 37 (2018).

26. Bensinger, S. J. et al. LXR signaling couples sterol metabolism to proliferation in the acquired immune response. Cell. 134, 97-111 (2008).

27. Schwartz, D. M. et al. JAK inhibition as a therapeutic strategy for immune and inflammatory diseases. Nat Rev Drug Discov. 16, 843-862 (2017). 
28. Shouda, T. et al. Induction of the cytokine signal regulator SOCS3/CIS3 as a therapeutic strategy for treating inflammatory arthritis. J Clin Invest. 108, 1781-1788 (2001).

29. Kinjyo, I. et al. Loss of SOCS3 in T helper cells resulted in reduced immune responses and hyperproduction of interleukin 10 and transforming growth factor-beta 1. J Exp Med. 203, 10211031 (2006).

30. Lewis, M. J. et al. Molecular Portraits of Early Rheumatoid Arthritis Identify Clinical and Treatment Response Phenotypes. Cell Rep. 28, 2455-24705 (2019).

31. Yamada, H. et al. Distinct features between HLA-DR + and HLA-DR- PD-1hi CXCR5- T peripheral helper cells in seropositive rheumatoid arthritis. Rheumatology (Oxford). 60, 451-460 (2021).

\section{Figures}

\section{Derivation cohort}

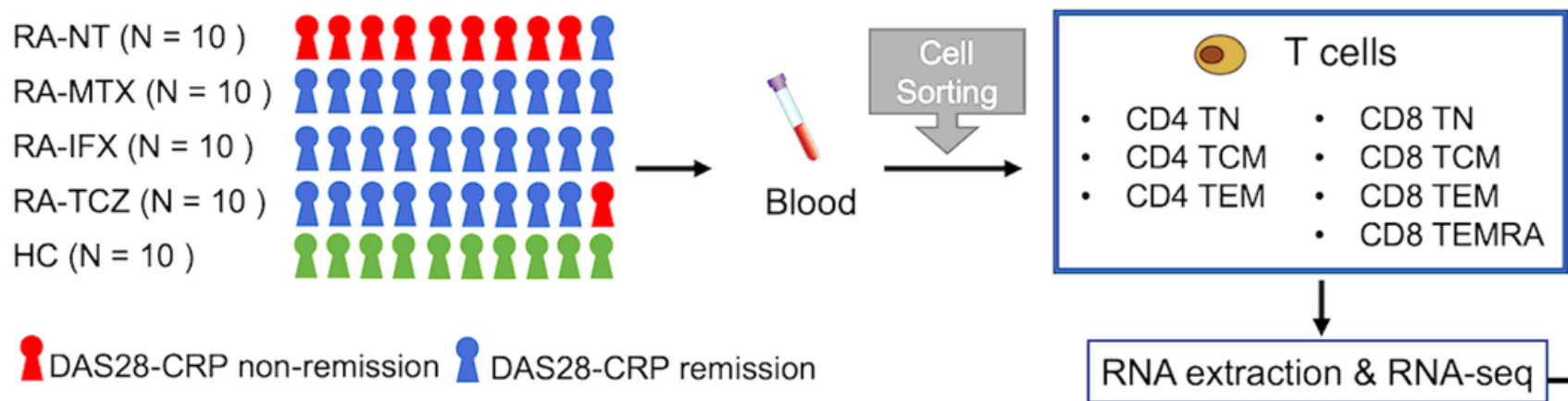

\section{Validation cohort}

RA-TCZ $(\mathrm{N}=6)$
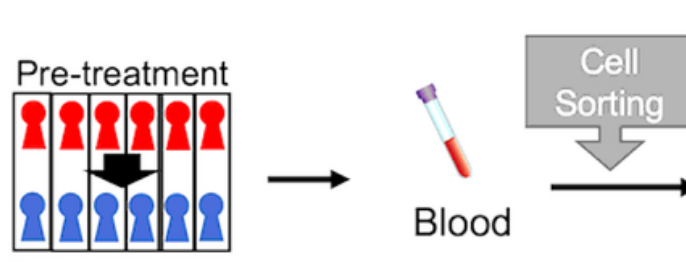

DAS28-CRP non-remission

DAS28-CRP remission

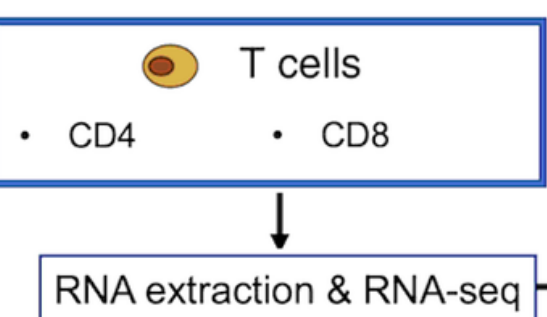

Analysis

Figure 1

Workflow of the current study. We used 2 cohorts: a derivation cohort including cross-sectional subjects and a validation cohort including longitudinal subjects treated by TCZ. In the derivation cohort, we measured gene expression according to cell subpopulations and summarized all CD4+ T cells and CD8+ $T$ cells in the expression analysis. HC, healthy control; IFX, infliximab; MTX, methotrexate; RA, rheumatoid arthritis; NT, non-treatment; TCM, central memory T cell; TCZ, tocilizumab; TEM, effector memory T cell; TEMRA, CD 45RA-positive effector memory $T$ cell; TN, naïve T cell. 
A
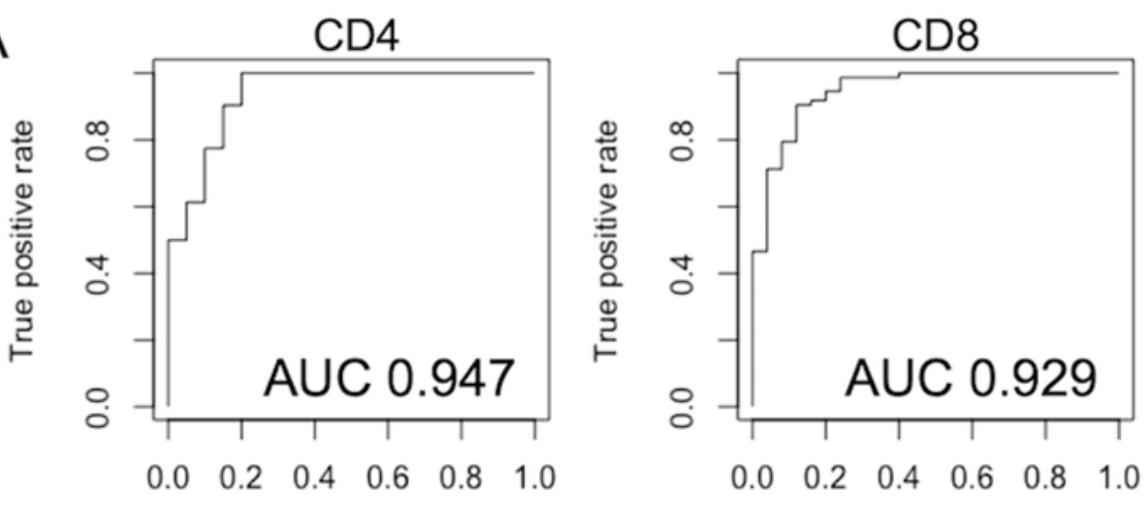

False positive rate

B

False positive rate

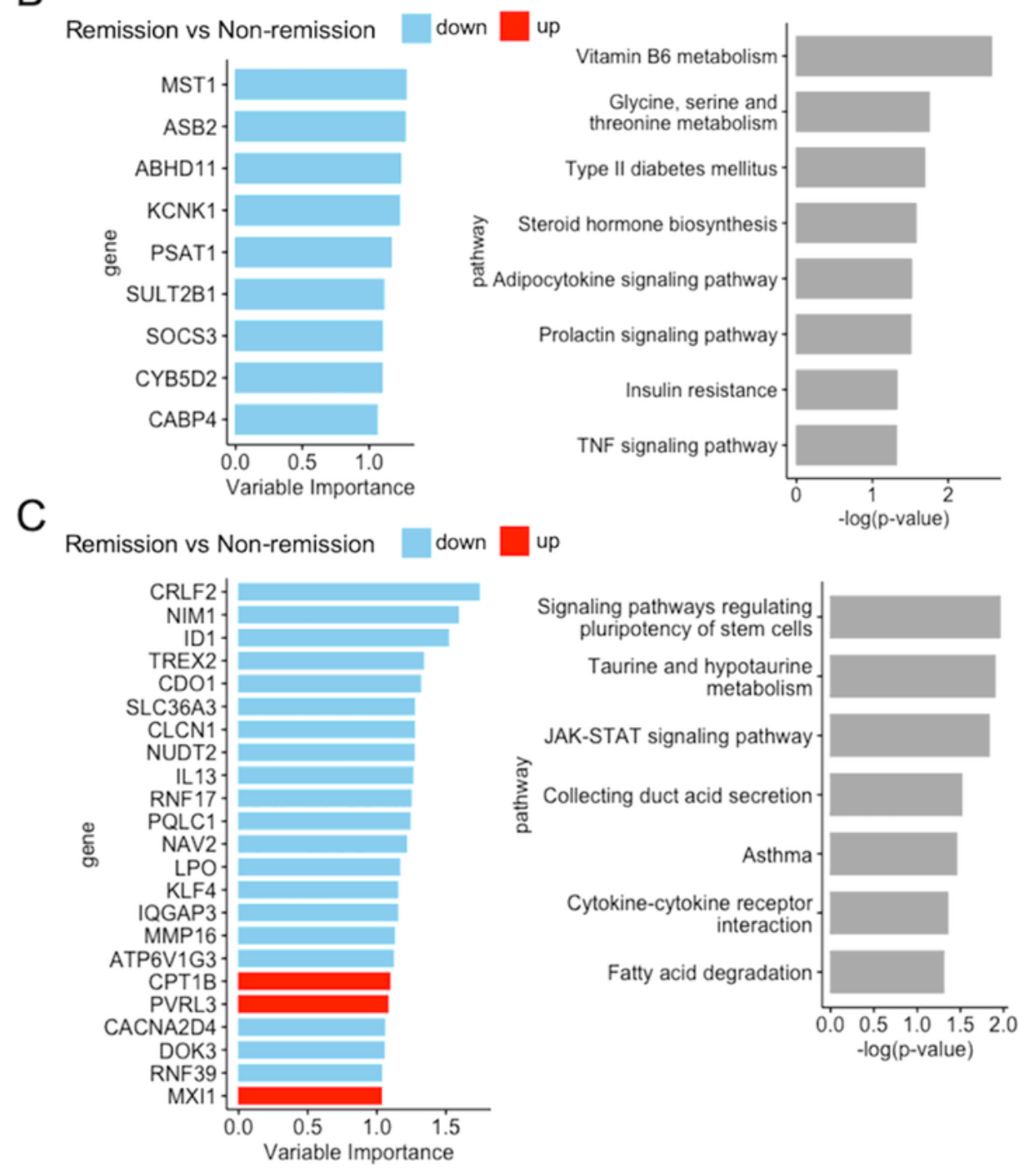

Figure 2

Identification of molecular signatures associated with remission in RA. A) ROC analysis according to the statistical model generated by the combination of lasso and PLS-R. B and C) Remission signature genes (left) and enrichment analysis (right) of CD4+ T cells and CD8+ T cells (B). The PLS-R analysis of gene expression, with blue indicating low and red toward high expression in remission patients. AUC, area 
under the curve; PLS-R, a partial least-squares regression; RA, rheumatoid arthritis; ROC, receiver operating characteristic.

A CD4TN

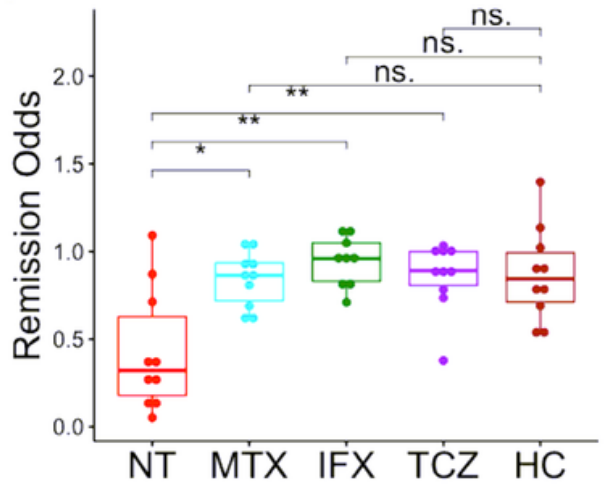

B CD4TN

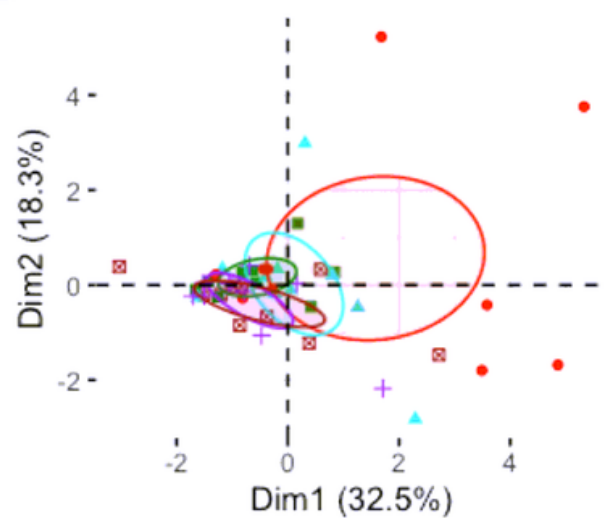

CD4TCM

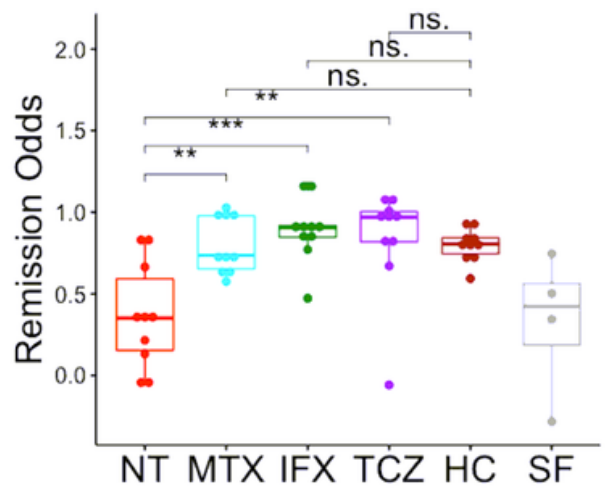

CD4TCM

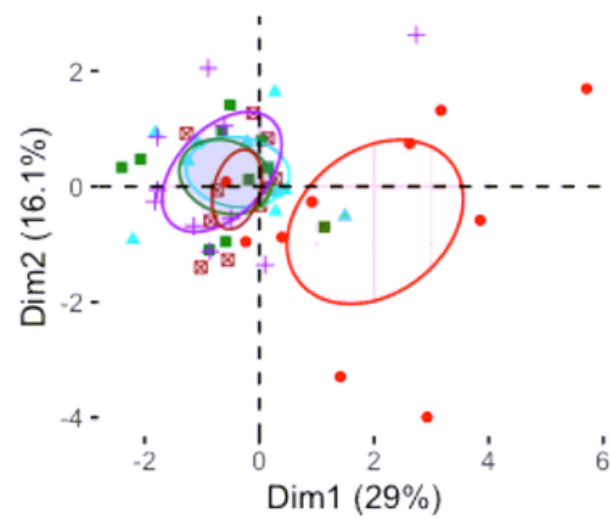

CD4TEM

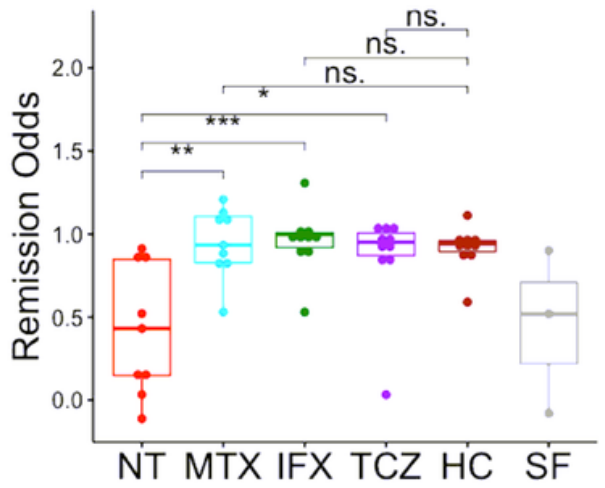

\section{CD4TEM}

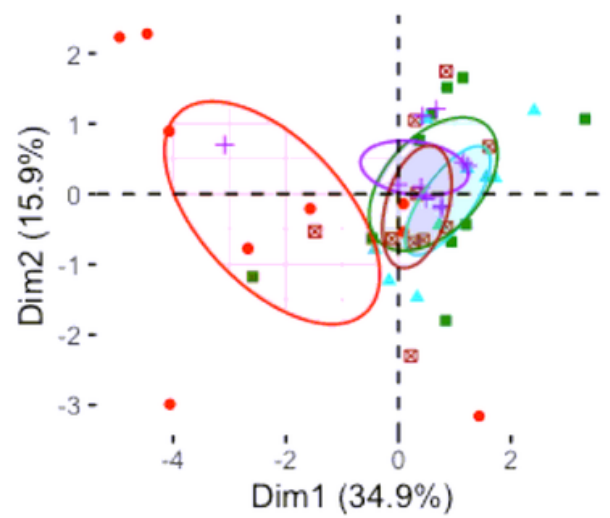

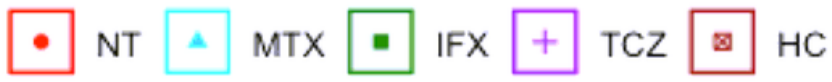

C $\quad$ CD4 (validation cohort)

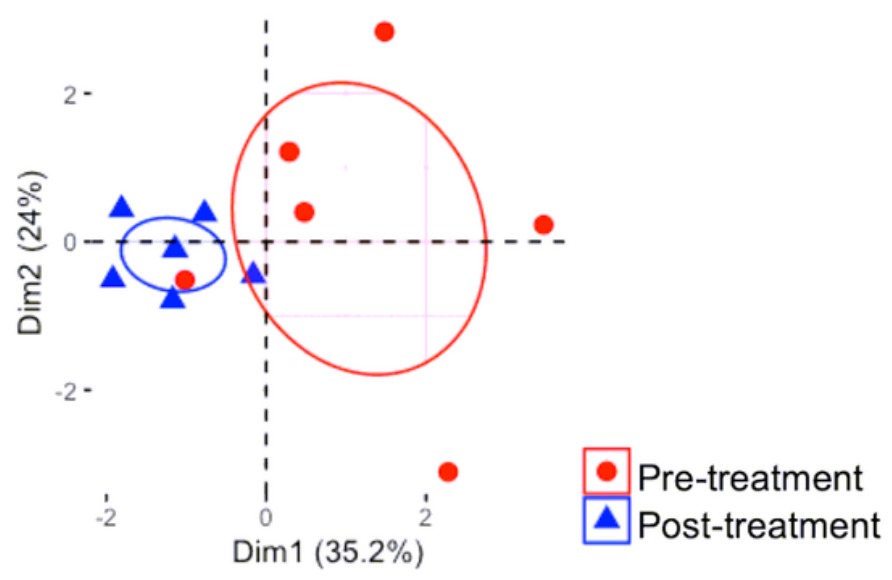

\section{Figure 3}

Remission odds and gene expression profiling of remission signature genes in CD4+ T cells. A)

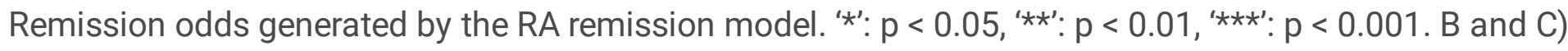
Principal component analysis using remission signature genes in the derivation cohort (B) and validation cohort (C). The ellipse shows the $95 \%$ confidence interval of the value of the principal component 
analysis. Patients in the validation cohort were treated by TCZ for 6 months and achieved remission after treatment. HC, healthy control; IFX, infliximab; MTX, methotrexate; RA, rheumatoid arthritis; NT, nontreatment; SF, synovial fluid; TCM, central memory T cell; TCZ, tocilizumab; TEM, effector memory T cell; TN, naïve T cell.
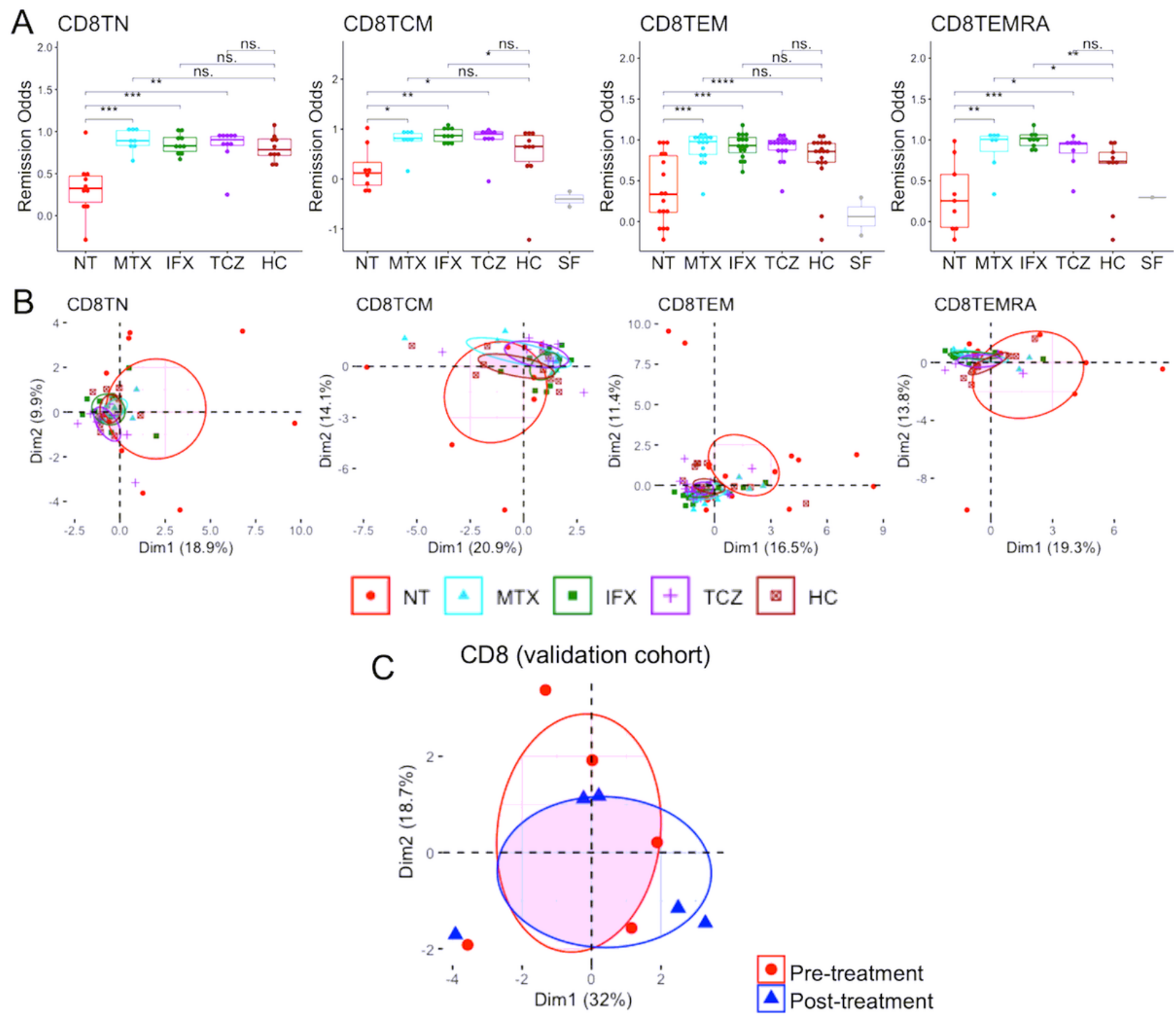

\begin{tabular}{|l|l}
- & Pre-treatment \\
\hline $\boldsymbol{\Delta}$ & Post-treatment
\end{tabular}

\section{Figure 4}

Remission odds and gene expression profiling of remission signature genes in CD8+ T cells. A)

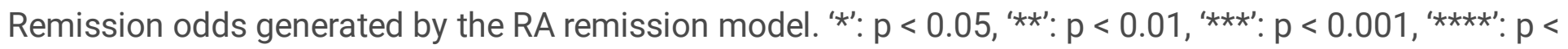
0.0001 . B and C) Primary component analysis using remission signature genes in the derivation cohort (B) and the validation cohort (C). The ellipse shows the $95 \%$ confidence interval of the value of principal component analysis. Patients in the validation cohort were treated by TCZ for 6 months and achieved remission after treatment. HC, healthy control; IFX, infliximab; MTX, methotrexate; RA, rheumatoid 
arthritis; NT, non-treatment; SF, synovial fluid; TCM, central memory T cell; TCZ, tocilizumab; TEM, effector memory T cell; TEMRA, CD45RA-positive effector memory T cell; TN, naïve T cell.

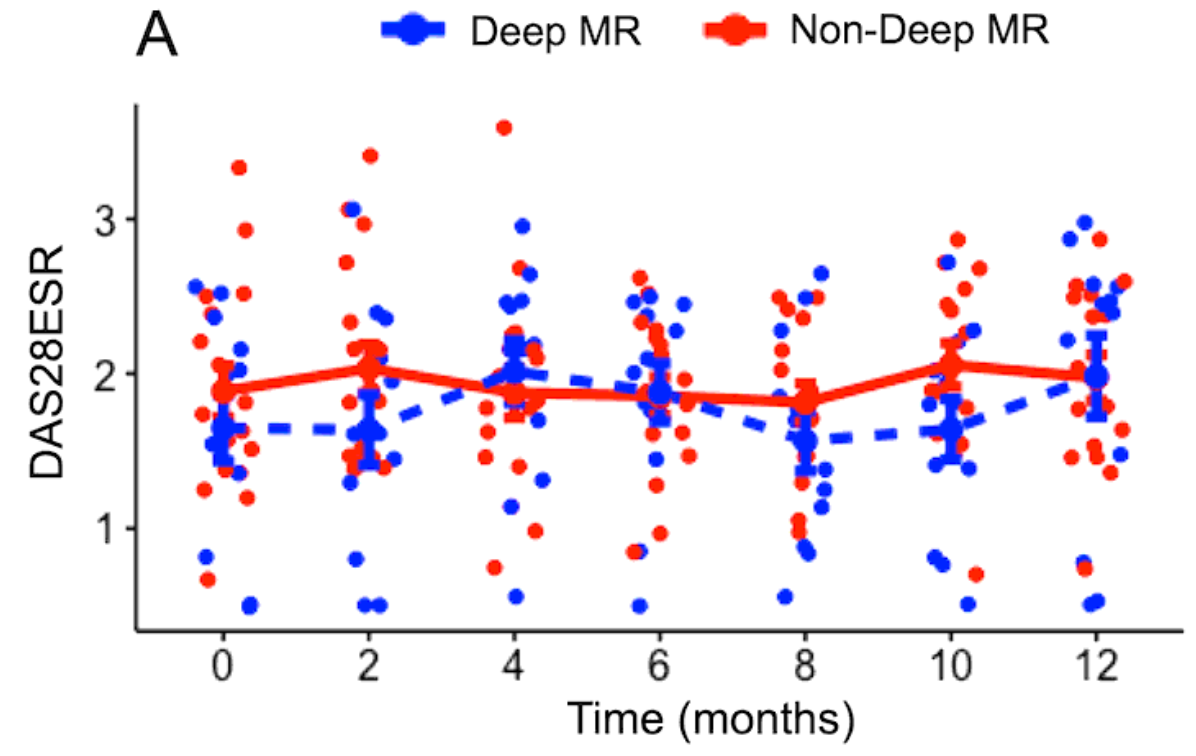

$\mathrm{B} \sim$ Deep MR Non-Deep MR

Figure 5

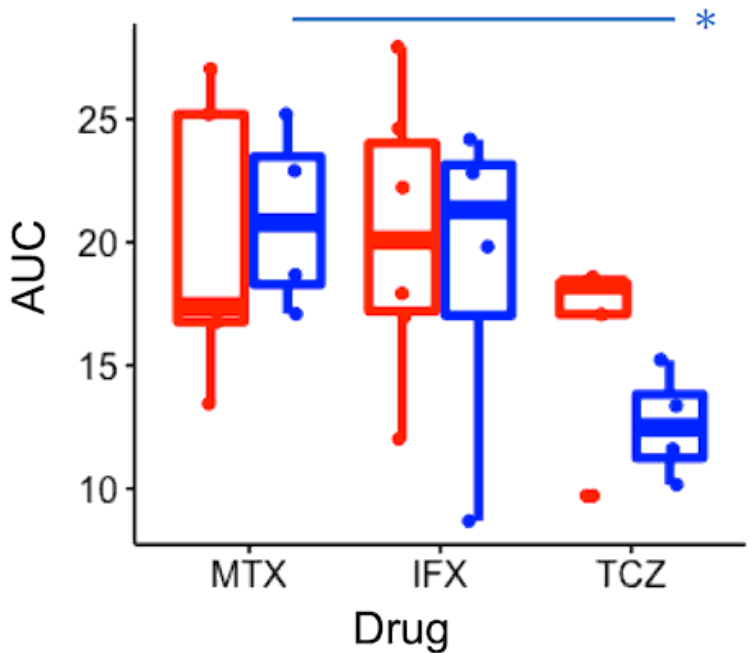

Relationship between MR and disease activity. A) The changes in DAS28-ESR during the follow-up period, which was split into two-week intervals. For each group, the average \pm standard error of DAS28-ESR within the same interval was shown by the line. B) Comparison of AUC (commutative DAS28-ESR during the 12-month follow-up) according to drug type. ' $*$ ': $p<0.05$. AUC, area under the curve; DAS28-ESR, disease activity score-28 ESR; IFX, infliximab; MTX, methotrexate; TCZ, tocilizumab.

\section{Supplementary Files}

This is a list of supplementary files associated with this preprint. Click to download.

- SuppleTableS12.docx

- SupplementaryTable3.xIsx 